\title{
Influencia del marketing estratégico en la internacionalización asociativa.
}

\section{Influence of strategic marketing planning for associative internationalization.}

\author{
Víctor Mideros \\ Universidad Técnica de Machala \\ vmideros1@utmachala.edu.ec \\ Machala - Ecuador \\ Michelle Guanaquiza \\ Universidad Técnica de Machala \\ mguanaqui1@utmachala.edu.ec \\ Machala - Ecuador \\ Patricia Micolta-Bagui \\ Universidad Técnica de Machala \\ pmicolta1@utmachala.edu.ec \\ Machala - Ecuador \\ Irene Sánchez-González \\ Universidad Técnica de Machala \\ isanchez@utmachala.edu.ec \\ Machala - Ecuador
}

\section{Resumen}

Objetivo: Analizar la influencia del marketing estratégico y la asociatividad de las empresas, como herramienta para la internacionalización. Métodos: Se utiliza el método descriptivo probabilístico, con enfoque transversal aplicando un cuestionario estructurado a una muestra de 400 gerentes de asociaciones de la provincia de El Oro. Resultados: El marketing estratégico y la cooperación de marketing, tienen un alto nivel de influencia sobre las empresas que se asocian para internacionalizarse. Conclusiones: Las asociaciones que cooperan entre sí, y que implementan gestiones de marketing estratégico en sus operaciones, tienen mayores resultados positivos para internacionalizar sus productos.

Palabras claves: Marketing estratégico, Cooperación de marketing, Internacionalización, Asociatividad, Asociaciones. 


\section{Abstract}

Objectives: Analyze the influence of strategic marketing and the associativity of companies, as a tool for internationalization. Methods: The descriptive-probabilistic method is used, with a cross-sectional approach, applying a structured questionnaire to a sample of 400 managers of associations in the province of El Oro. Results: Strategic marketing and marketing cooperation have a high level of influence on companies that associate to internationalize. Conclusions: Associations that cooperate with each other, and that implement strategic marketing efforts in their operations, have greater positive results to internationalize their products.

Keywords: Strategic marketing, Marketing cooperation, Internationalization, Associativity, Associations.

\section{Introducción}

La internacionalización exportadora de una empresa es el resultado directo de su éxito, lo cual solo es posible creando estrategias de mercadotecnia y de comercio internacional (Álvarez et $a l ., 2017)$ que las proporcionan el conocimiento de marketing estratégico y comunitario.

Conceptualizando el marketing estratégico (Hernandez, 2018), para la internacionalización asociativa, una empresa deberà seguir los siguientes pasos: explorar y buscar nuevos nichos de mercado; paralelamente es necesario que se identifique segmentos potenciales de mercado a nivel internacional;valorar el potencial e intereses de esos mercados, allende a nuestras fronteras; orientar a las empresas por medio de consultorías en esta búsqueda de oportunidades; y finalmente diseñar un adecuado y técnico plan de acción.

El mundo globalizado demanda una mayor coordinación y organización interna y externa de sus unidades productivas (Aguilar, 2017), y esto deberà producir estrategias de mejoramiento de los sistemas y subsistemas productivos. En este procedimiento se van a encontrar dos escenarios sinérgicos que consisten en el apoyo productivo empresarial y de marketing y,el escenario regulatorio de aranceles en el país destino de dichos productos. En este último enunciado muchas veces se conjugan los intereses de empresas privadas o mixtas , o del sector gubernamental, y por otra parte el estado creando políticas de exportación reales y justas y llegando a acuerdos con gobiernos amigos para obtener tasas arancelarias beneficiosas. La capacidad asociativa es lo que permite internacionalizarse a las empresas y es un factor potencial que estimula a los empresarios a desarrollarse. (Alarcón, 2018).

La historia del marketing en lo referente a comercio internacional ha evolucionado, impulsando cambios adaptativos (Lobos, 2019). La internacionalización es eficiente sólo cuando se utilizan las estrategias de marketing (Sánchez-González, et al., 2018)para lograr el éxito expansivo de las empresas (Mosquera, 2018). Extrapolando lo dicho anteriormente en la realidad ecuatoriana en lo concerniente a la Provincia de El Oro y específicamente en los sectores Agrícola y Acuícola, (Bustamante, 2017), los empresarios han cumplido con las siguientes estrategias de 
marketing para la internacionalización asociativa:Desde principios de los años 50 del siglo pasado existía un solo monopolio en el país que lo ejercía la empresa multinacional Del Monte, con su subsidiaria local la "Bonita Banana". A medida que se fueron creando empresas de capital privado se alternò el mercado norteamericano con el europeo, sobre todo el mercado alemán e italiano lo que produjo una expansión muy potente de la producción bananera en el Ecuador que ha ayudado a la comunidad orense y ecuatoriana en general, así como actualmente a la agroindustrial con la venta de harina de banano, purè de banano, yogurt de banano, etc.

Lo mismo se dió en el sector acuicultor que ahora es una gigante fuente de ingreso en el Ecuador y cuyas ventas se han diversificado desde los Estados Unidos, hasta Alemania, Francia y a nuestro mayor comprador que es la China. Actualmente se han identificado nuevos segmentos de mercado como lo es el gigante mercado ruso. Se ha valorado la calidad de nuestro banano y camarón en dichos mercados ya que al tener el Ecuador la mayor cantidad de días-sol del mundo por años nuestros productos son los mejores de todo el orbe.

Ecuador empleó estrategias muy inteligentes como la contratación de consultorías allende a nuestras fronteras y la colocación de stands informativos en las principales ferias informativas del mundo que están en EEUU, Alemania, Francia, Bélgica, etc. El plan de acción de nuestra exportación y riqueza bananera se basa sobre todo en la empresa privada la que recibe fertilizantes como la urea a menor precio, aéreo fumigación subvencionada, control de calidad en el campo y la creación de muchas empresas exportadoras directas para evitar los monopolios.

Actualmente se ha visto también la creación de cooperativas agrícolas medianas y pequeñas, aunque ellas al igual que los pequeños productores reciben un trato comercial injusto en las políticas de precios de parte de los monopolios. (Orozco, 2017)

\section{Marco Teórico}

\section{Planeación estratégica}

La planeación estratégica es el proceso que sirve para formular y ejecutar las estrategias de la organización con la finalidad de insertarla, según su misión, en el contexto en el que se encuentra. (Chiavenato, 2017)

La planeación estratégica también se la conoce como una herramienta de dirección que permite a una organización prepararse y definir la forma de conseguir su objetivo para enfrentarse a los desafíos futuros de su entorno, orientando sus acciones hacia metas realistas y tomando decisiones estratégicas para el éxito de la compañía. (Bernal, 2018)

\section{La cooperación de marketing en las asociaciones del Ecuador en la provincia de El Oro dentro de los sectores acuícolas y agrícolas.}

Para todas las empresas y asociaciones es importante mantener un grado alto de posicionamiento en el mercado con sus productos, con el objetivo principal de maximizar sus 
ventas y obtener buenas ganancias; es por eso que una buena planeación estratégica de marketing se hace indispensable para estas asociaciones, buscando el bienestar del consumidor a través de la presentación de productos que satisfagan sus necesidades.

La planeación de marketing tiene un alto aporte en el funcionamiento de las asociaciones para la comercialización eficaz de cualquier producto, manteniendo objetivos fijos a alcanzar. Este es el proceso mediante el cual se visualiza el comportamiento del futuro de la organización (Moreno, 2017)

Las estrategias de marketing son aquel conjunto de actividades encaminadas a la planificación y análisis del entorno del mercado, así mismo el marketing estratégico analiza las variables del marketing mix (Hartline, 2014). Es decir, que se incluye el conocimiento del producto y sus características, precio acorde al análisis del mercado, la plaza que es el análisis del entorno del mercado y la promoción que las empresas deben definir y planificar bien esto refleja buenos resultados para la empresa.

\section{Asociatividad}

La asociatividad según Vélez et al. (2019), es un método utilizado por las organizaciones para unir voluntades, recursos con iniciativas que impulsen a un beneficio en común, dando apertura a la competitividad dentro del mercado globalizado con estrategias específicas para la presentación de productos diferenciales.

Todas las empresas públicas o privadas, se destacan por realizar actividades que permitan suplir una necesidad en el mercado, por tal motivo se asocian mientras perciban un beneficio de manera conjunta, en donde buscan compartir valores y habilidades entre sí (Vélez et al., 2019). Una estrategia clave para el desarrollo de las MIPYMES en el mundo globalizado es fomentar la asociatividad de las empresas, promoviendo la creación de clusters y redes empresariales en cadenas productivas competitivas. (Bada, Rivas y Littelewood, 2017)

Una de las principales estrategias que se aplican para optimizar recursos, aumentar nivel de ventas y fomentar las interrelaciones comerciales, es la asociatividad, a través de la formación de grupos de afinidad, gremios y sobretodo la implementación de redes de cooperación entre negocios o empresas con algún tipo de afinidad, especialmente por el tipo de actividad a la que se dedican. (Fajardo et al., 2016)

\section{Intensidad de internacionalización}

En el proceso de expansión y crecimiento de una pyme, tomar la decisión de internacionalizarse conlleva una serie de análisis que inducen a la concertación y a la preparación previa de la empresa para luego ejercer un compromiso institucional que involucra visión, objetivos estratégicos, dependencias y recursos, tanto humanos como productivos, tecnológicos, capital intelectual, activos intangibles, entre otros. (Hoyos, 2019)

El concepto de internacionalización de las empresas está asociado al conjunto de operaciones que facilitan vínculos estables entre los negocios y el mercado internacional a lo largo de 163 
procesos que incrementan el compromiso y la proyección internacional, (Novoa, 2016). En base a lo mencionado por Novoa en su Artículo "La internacionalización de las empresas en Colombia", en las últimas décadas América Latina ha entrado en un auge de expansión empresarial internacional, permitiendo que algunas empresas latinoamericanas se establezcan en estos mercados con pie firme; pero esto no ha sido nada fácil debido a los procesos y etapas que se deben seguir para esto, por lo cual (Novoa, 2016) señala lo siguiente; "Los países latinoamericanos presentaron una dinámica importante de apertura al comercio internacional, especialmente a partir del año 2010. El contexto económico se caracterizaba por la revaluación y los buenos precios de los commodities. Las multilatinas mejoraron su rol en el contexto internacional, sin descuidar su mercado de origen".

El papel importante de las empresas en el mercado exterior no puede ser una casualidad, las estrategias de cooperación por medio de la asociatividad son fundamentales ya que las alianzas estratégicas entre empresas ayudan a aliviar el peso de lo difícil que podría ser la internacionalización. "La internacionalización resulta especialmente crítica para la mediana empresa familiar dado que se trata de un proceso difícil y exigente que requiere de recursos humanos financieros y logísticos, así como de una clara estrategia para avanzar". (Andrade, 2017)

El modelo de Uppsala, esta teoría secuencialista afirma que las decisiones de internacionalización de las empresas se ven limitadas por dos factores: la información y los recursos. Es decir, los principales obstáculos para abordar los mercados exteriores proceden de la carencia de información (lo que genera incertidumbre) y de la escasez de recursos para afrontar el proceso. (Rave et al., 2018). Para estudiar el resultado de la internacionalización de las empresas se requiere analizar tanto los factores internos como los externos. ( Botello, 2014)

Ecuador es el mayor exportador de banano del mundo, el segundo exportador de camarón y atún, y el tercer exportador de flores. Es decir, aún hay oportunidades para aprovechar más estos productos tradicionales. También hay oportunidades para aquellos nuevos productos con potencial exportable. Entre éstos se destacan: la balsa, el brócoli y la teca, que ya se están exportando, los granos andinos (y productos derivados) como la quinua, el chocho y el amaranto; las frutas andinas (tomate de árbol, uvilla y mora), además de la merluza y la cobia, entre los pescados con potencial exportable. ( MAGAP, 2016).

\section{Producción y Exportación agrícola en la Provincia de El Oro.}

La producción bananera según la Organización de las Naciones Unidas para la Alimentación y la Agricultura (2020), es uno de los cultivos más rentables y extensos en América Latina y el Caribe, además es el principal rubro de ingresos económicos de exportación agrícola del Ecuador, su demanda se basa en la calidad, de esta forma se ha convertido en una fruta muy consumida en muchos países, debido a sus propiedades nutricionales, constituidas principalmente por macro y micronutrientes, posee también propiedades fitonutritivas y compuestos bioactivos que refuerzan la salud, es un sustento vital para las familias de la región costa del Ecuador. Las perspectivas de crecimiento a nivel mundial, especialmente de Ecuador, principal exportador de la fruta en el mundo, es alta. (Zhuminaicela, et al., 2020). 
Ecuador tiene alrededor de 162.236 hectáreas sembradas de Banano y cuenta con 4.473. El $78 \%$ de los productores de banano del país son de pequeñas empresas, y si se suma a los medianos se alcanza el 95.6\%. En este sentido, la producción del banano en el país gira principalmente en el ámbito de la economía familiar y la Economía Popular y Solidaria (Ministerio de Comercio Exterior, 2017).

Los productores de banano se concentran principalmente en las provincias de El Oro, Guayas y Los Ríos, las mismas que abarcan el $41 \%, 34 \%$ y $16 \%$ de los productores, respectivamente. En la provincia de El Oro se sitúan la mayor parte de lo pequeños productores de banano del país (aproximadamente 42\%), mientras que los grandes productores principalmente en las provincias de Guayas y Los Ríos. ( Ministerio de Comercio Exterior, 2017).

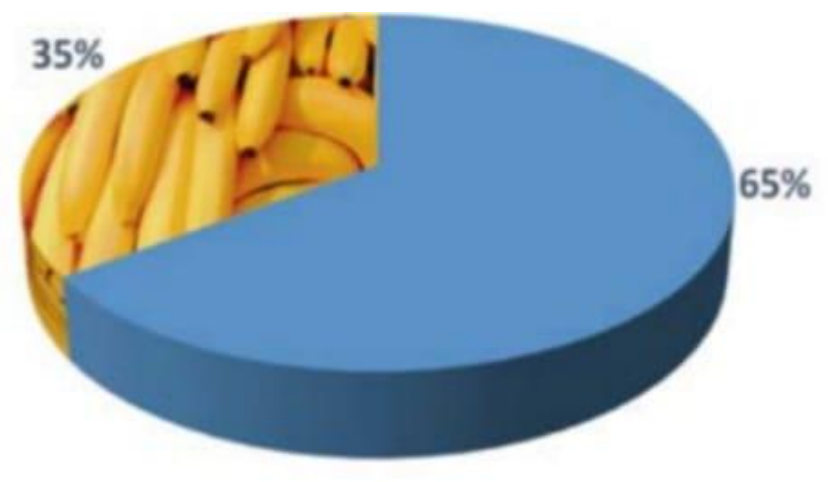

Fuente: Ministerio de comercio exterior.

Uno de los ejemplos más destacados de pequeños productores es la Asociación de Pequeños Productores Bananeros "El Guabo", creada en 1997. El Guabo es una asociación de pequeños productores de las provincias de Azuay, El Oro y Guayas. Hoy en día, El Guabo cuenta con 350 miembros y emplea a más de 2.000 personas. El Guabo tiene como objetivo principal satisfacer las demandas de sus clientes con productos de alta calidad, bajo el sistema de comercio justo, con el objetivo de mejorar las condiciones de sus socios junto con su entorno social y laboral. ( Ministerio de Comercio Exterior, 2017)

Esta Asociación ha establecido su propio sistema de monitoreo que incluye el tratamiento de la fruta de la granja al puerto. Esto ha dado como resultado la eficiente producción y exportación de: Banano de Bebé (CJ), Banano Orgánico, Banano Tipo Cavendish Valery y Puré de banano. ( Ministerio de Comercio Exterior, 2017)

En 2007, "El Guabo" fue uno de las asociaciones más importantes representando el 33\% de la cantidad total exportada por este sector. En 2015, las exportaciones fueron de USD 241 millones, mientras que en 2016 las exportaciones alcanzaron USD 304 millones. (Ministerio de Comercio Exterior, 2017) 


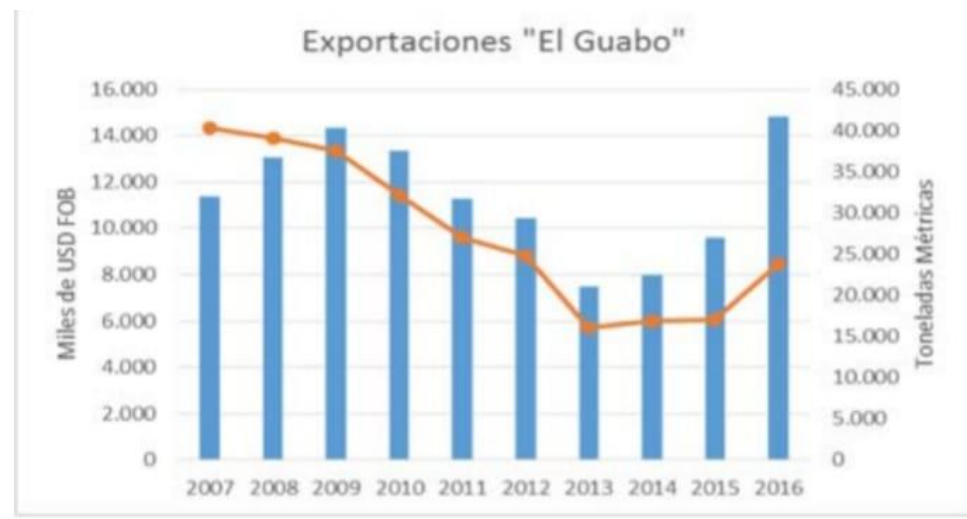

Fuente: Ministerio de comercio exterior.

\section{Producción y Exportación Acuícola en la Provincia de El Oro.}

A nivel mundial, las estadísticas de pesca y acuicultura de FAO dan cuenta que la producción acuícola global registró un crecimiento promedio anual de $8.2 \%$ en el período 1950-2015, para llegar a 106 millones de toneladas métricas (TM) en 2015, reflejando una significativa tendencia ascendente que le permitió aportar con más de la mitad de las especies acuáticas para consumo humano mundial en 2014, en comparación con la participación de $7 \%$ que tenía cuarenta años atrás. (ESPAE, ESPOL, 2018).

Cifras de FAO dan cuenta que la producción resultante de la acuicultura en Ecuador inicia sus registros en 1969 y está constituida básicamente por la cría de camarones , que sumó 403 mil TM en 2015, lo que representó 94.5\% de la producción acuícola total en el país. La diferencia comprendía 17.4 mil TM de tilapia del Nilo, 4.5 mil TM de trucha arcoíris y valores menores de otras 4 especies. ( ESPAE, ESPOL, 2018).

Información publicada por el Servicio de Rentas Internas (SRI) sobre variables por actividad económica en el país señala que en el año 2016 las actividades relacionadas con la industria de producción acuícola en Ecuador registraron un nivel combinado de ingresos por USD 2,449.6 millones, que significó una variación anual de $21 \%$, con exportaciones netas que representan el 38\% de los ingresos totales. (ESPAE, FAO, 2018).

El sector costero de la provincia de El Oro, es característicamente fructífero y productivo, donde prevalecen los factores diferenciadores propios de la región, conformada por esteros y manglares cuya diversidad natural del suelo y riqueza mineral de sus aguas facilitan en gran medida el cultivo, la pesca y comercialización del camarón cultivados en los cantones y parroquias dedicados a esta actividad. Por tal motivo, los actores principales inmersos en el contexto del cultivo de camarón radicados en la provincia de El Oro cronológicamente tienen este en factor-tiempo un punto a su favor, el cual les permite diferenciarse de sus inmediatos competidores y afrontar los desafíos de la globalización en el escenario internacional. ( Valera, Elizalde, Solórzano y Valera, 2017). 
Según el Instituto Nacional de Pesca dentro del perfil costanero ecuatoriano, particularmente en la provincia de El Oro las principales especies de camarón blanco, café y rojo; cultivados y capturados existen en la siguiente variedad: Protrachypene precicua (Camarón pomada), Litopenaeus vannamei (Camarón blanco tipo langostino), Litopenaeus stylirostris (Camarón blanco tipo langostino), Litopenaeus occidentalis (Camarón blanco tipo langostino), Farfantepenaeus californiensis (Camarón café tipo langostino). Cuyas características distintivas y de estándar mundial se justifican en su textura, tamaño y sabor, cualidades del camarón que resultan óptimas para su comercialización nacional e internacional, cumpliendo con los requerimientos del bloque comercial europeo. (Valera, Elizalde, Solórzano y Valera, 2017).

En este contexto se puede afirmar que las condiciones de producción camaronera en la Provincia de El Oro tiene oportunidades favorables para seguir ampliando el mercado internacional de este rubro con mayor firmeza. Respecto de la internacionalización la literatura dice lo siguiente: "Internacionalización es la fase en la cual los países se especializan en la producción de bienes y servicios en compensación a la exportación de estos productos, a través de las ventajas competitivas de cada país, dando origen a la internación de compañías mediante las ventajas competitivas U por medio de su participación en el mercado internacional a través de las diversas actividades internacionales que generan diferentes tipos de resultados como: comercial, financiero y de conocimiento. ( Valera, Elizalde, Solórzano y Valera, 2017).

De acuerdo a lo expuesto por los autores anteriores la asociatividad de las empresas como de los pequeños productores en la Provincia han contribuido grandemente para efectivizar la exportación camaronera; así lo manifiesta el siguiente enunciado. "La producción y exportación del camarón retribuye sustancialmente en los rubros económicos y actividades comerciales realizadas dentro del territorio nacional, la región y particularmente en la provincia de El Oro. En consecuencia, 200 mil familias dependen de la producción camaronera en el Ecuador, además de otros empleos vinculados en el contexto de su cultivo correspondientes a: laboratorios de larvas, procesadoras de balanceado, insumos, empacadoras, logística y transporte, y otros servicios conexos que en conjunto permiten a los productores locales y nacionales, focalizar y dinamizar su internacionalización. ( Valera et al., 2017).

\begin{tabular}{|l|c|c|}
\hline Provincias & $\begin{array}{l}\text { Acuicultura y pesca de } \\
\text { camarón }\end{array}$ & $\begin{array}{l}\text { Porcentaje de } \\
\text { Participación }\end{array}$ \\
\hline EL ORO & 147.977 & $37,68 \%$ \\
\hline ESMERALDAS & 3.113 & $0,79 \%$ \\
\hline GUAYAS & 169.124 & $43,06 \%$ \\
\hline MANABI & 56.067 & $14,28 \%$ \\
\hline GALAPAGOS & 97 & $0,02 \%$ \\
\hline SANTA ELENA & 16.379 & $4,17 \%$ \\
\hline VALOR AGREGADO BRUTO & 392.757 & $100,00 \%$ \\
\hline
\end{tabular}

Fuente: Ministerio de comercio exterior. 


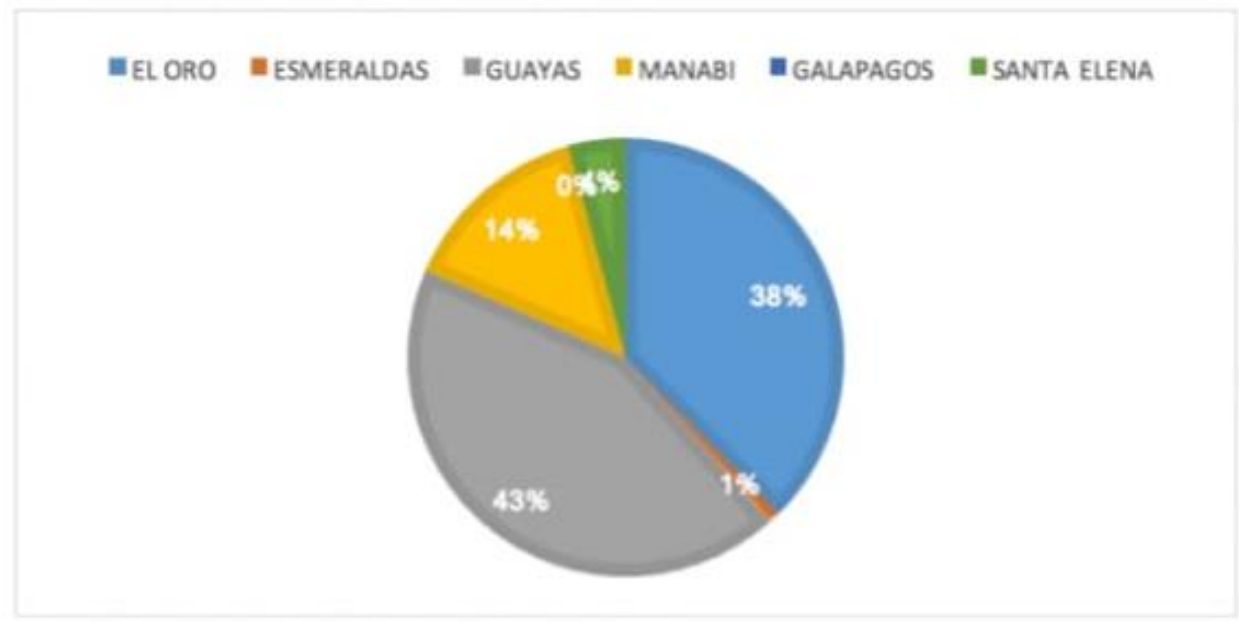

Fuente: Ministerio de comercio exterior.

\section{Materiales y métodos}

En la presente investigación se hizo uso del método cuantitativo, el cual mediante encuestas permitió recopilar información de 400 gerentes de asociaciones de la provincia de El Oro.

La investigación se aplicó a un universo conformado por 1200 asociaciones, pertenecientes a las industrias con mayor actividad (agricultura, acuicultura y pesca), de las cuales se depuraron 400 asociaciones que exportan y que no exportan sus productos, pero tienen el potencial para desarrollar este tipo de actividades al mercado internacional. Dentro de las 400 asociaciones se encuentran 1121 empresas, las cuales fueron distribuidas en función a la cantidad de estudiantes de los horarios nocturno y matutino, 75 estudiantes en total. Se aplicó el método probabilístico con encuestas y escalas de Likert para la recolección de información, que se levantó desde diciembre del 2019 hasta febrero del 2020.

\section{Resultados y discusión}

De acuerdo a las encuestas realizadas en las diferentes empresas de asociaciones pertenecientes a las industrias con mayor actividad (agricultura y acuicultura), se pudieron obtener diferentes resultados enfatizando las variables de planeación estratégica, asociatividad, cooperación de marketing e intensidad de internacionalización.

Como se puede observar en la tabla 1 , los socios manifestaron que se encuentran totalmente de acuerdo en que la planeación es un proceso fundamental para el funcionamiento de la empresa, y por ello se plantean estrategias para alcanzar los objetivos propuestos por la empresa. Así mismo, los socios están de acuerdo con plantearse objetivos de manera periódica dentro de la empresa y con la adquisición de nuevos equipos. 
Conforme a la tabla 2, los socios se encuentran totalmente de acuerdo en que las estrategias de negociación se desarrollen en escenarios de ganar-ganar y establecer prácticas comerciales y leales con los socios, así mismo piensan que es fundamental la ética en los negocios y afectos con los empresarios de la competencia. Desde este punto los socios sienten que es muy importante relacionarse, así como interesarse y conocer estrategias de asociaciones, cadenas o redes.

En la tabla 3, se puede analizar que la mayor parte de los asociados se encuentran de acuerdo con ferias, promociones y misiones comerciales y una gran minoría está en desacuerdo por esto, por otro lado, las asociaciones se encuentran de acuerdo con delegaciones de búsqueda de nuevos clientes, así como lograr compartir información y hacer investigaciones de mercados que permitan el desarrollo de nuevos productos.

Por último en la tabla 4, para la mayor parte de la asociaciones en la intensidad de internacionalización tienen un número bajo de mercados extranjeros que son mercados objetivo de las empresas pertenecientes a las mismas, también se resalta una baja intensidad para la participación de ventas en exportaciones, acuerdos de cooperación formales con socios extranjeros y para la cantidad de redes internacionales en las que participa la asociación.

\section{Tabla 1 (Planificación estratégica)}

\begin{tabular}{|l|l|c|c|c|c|c|}
\hline \multicolumn{2}{|c|}{$\begin{array}{l}\text { PLANEACIÓN } \\
\text { ESTRATÉGICA }\end{array}$} & $\begin{array}{c}\text { Totalmente en } \\
\text { desacuerdo }\end{array}$ & $\begin{array}{c}\text { En } \\
\text { desacuerdo }\end{array}$ & Neutral & $\begin{array}{c}\text { De } \\
\text { acuerdo }\end{array}$ & $\begin{array}{c}\text { Totalmente } \\
\text { de acuerdo }\end{array}$ \\
\hline PE1 & $\begin{array}{l}\text { La planeacion es un } \\
\text { proceso fundamental } \\
\text { para el funcionamiento } \\
\text { de la empresa. }\end{array}$ & 5 & 18 & 119 & 227 & 274 \\
\hline PE2 & $\begin{array}{l}\text { Se plantea objetivos de } \\
\text { manera periódica } \\
\text { dentro de la empresa. }\end{array}$ & 3 & 14 & 108 & 269 & 252 \\
\hline PE3 & $\begin{array}{l}\text { Se plantean estrategias } \\
\text { para alcanzar los } \\
\text { objetivos propuestos } \\
\text { por la empresa. }\end{array}$ & 7 & 15 & 117 & 236 & 271 \\
\hline PE4 & $\begin{array}{l}\text { Adquisición de nuevos } \\
\text { equipos. }\end{array}$ & 19 & 37 & 152 & 227 & 209 \\
\hline
\end{tabular}

\section{Ilustración 1 (Planificación estratégica)}

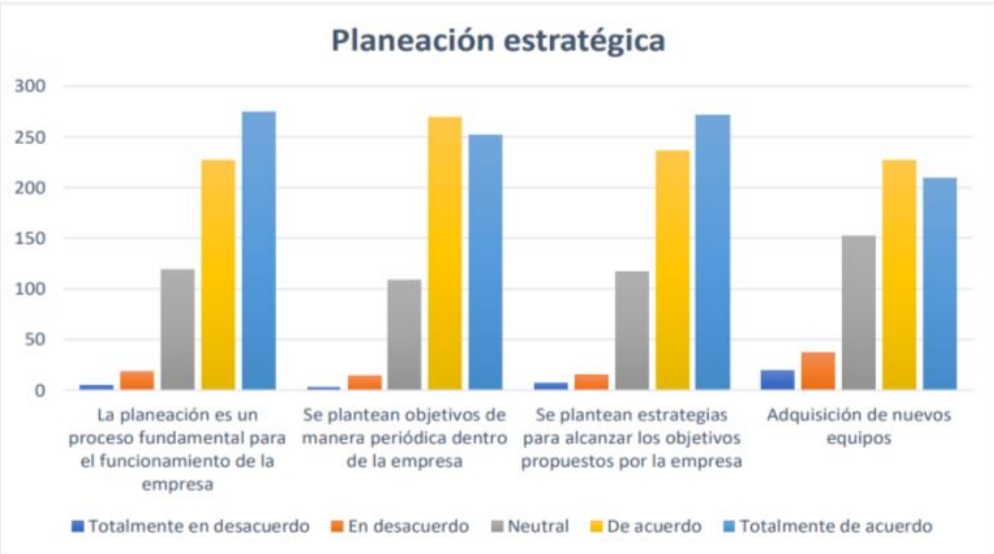




\section{Tabla 2 (Asociatividad)}

\begin{tabular}{|l|l|c|c|c|c|c|}
\hline \multicolumn{2}{|c|}{ ASOCIATIVIDAD } & $\begin{array}{l}\text { Totalmente en } \\
\text { desacuerdo }\end{array}$ & $\begin{array}{c}\text { En } \\
\text { desacuerdo }\end{array}$ & Neutral & $\begin{array}{c}\text { De } \\
\text { acuerdo }\end{array}$ & $\begin{array}{c}\text { Totalmente } \\
\text { de acuerdo }\end{array}$ \\
\hline AS1 & $\begin{array}{l}\text { Las estrategias de } \\
\text { negociacion se } \\
\text { desarrollan en } \\
\text { escenarios de ganar- } \\
\text { ganar }\end{array}$ & 7 & 28 & 92 & 221 & 297 \\
\hline AS2 & $\begin{array}{l}\text { Se manifiesta voluntad } \\
\text { de asociación }\end{array}$ & 7 & 16 & 122 & 241 & 260 \\
\hline AS3 & $\begin{array}{l}\text { Se establecen buenas } \\
\text { practicas comerciales y } \\
\text { leales con los asociados }\end{array}$ & 2 & 23 & 107 & 251 & 263 \\
\hline AS4 & $\begin{array}{l}\text { Hay ética en los } \\
\text { negocios }\end{array}$ & 4 & 16 & 98 & 228 & 300 \\
\hline AS5 & $\begin{array}{l}\text { Hay afectos con los } \\
\text { empresarios de la } \\
\text { competencia. }\end{array}$ & 8 & 18 & 170 & 249 & 197 \\
\hline AS6 & $\begin{array}{l}\text { Hay interés por } \\
\text { alianzas estratégicas } \\
\text { con competidores. }\end{array}$ & 15 & 19 & 169 & 245 & 197 \\
\hline AS7 & $\begin{array}{l}\text { Conoce y le interesa } \\
\text { estrategias de } \\
\text { asociacion, cadenas o } \\
\text { redes }\end{array}$ & 10 & 13 & 157 & 247 & 218 \\
\hline AS8 & $\begin{array}{l}\text { Indique cual es el } \\
\text { grado de importancia } \\
\text { de asociarse para su } \\
\text { empresa. }\end{array}$ & 3 & 12 & 96 & 237 & 297 \\
\hline
\end{tabular}

\section{Ilustración 2 (Asociatividad)}

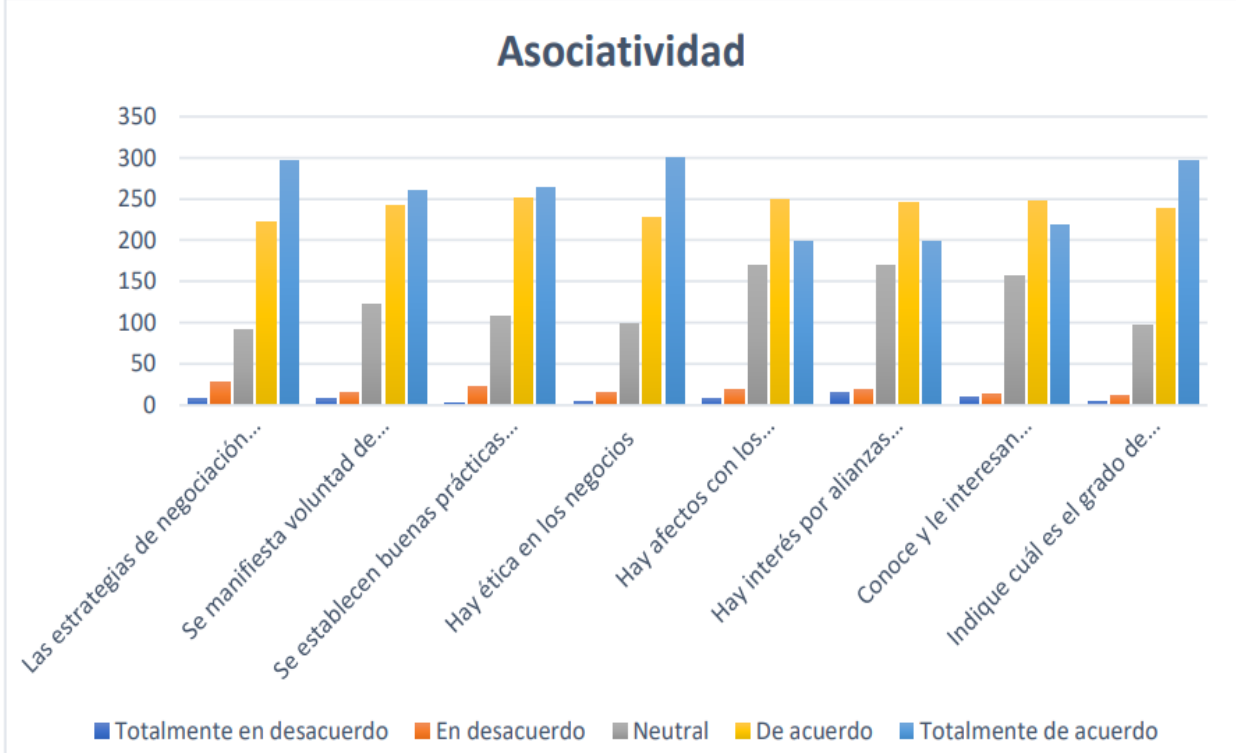


Tabla 3 (Cooperación de marketing)

\begin{tabular}{|l|l|c|c|c|c|c|}
\hline \multicolumn{2}{|c|}{$\begin{array}{l}\text { COOPERACIÓN DE } \\
\text { MARKETING }\end{array}$} & $\begin{array}{l}\text { Totalmente en } \\
\text { desacuerdo }\end{array}$ & $\begin{array}{c}\text { En } \\
\text { desacuerdo }\end{array}$ & Neutral & $\begin{array}{c}\text { De } \\
\text { acuerdo }\end{array}$ & $\begin{array}{c}\text { Totalmente } \\
\text { de acuerdo }\end{array}$ \\
\hline CP1 & $\begin{array}{l}\text { Ferias y promociones } \\
\text { comerciales }\end{array}$ & 108 & 34 & 150 & 188 & 166 \\
\hline CP2 & Misiones comerciales & 95 & 77 & 140 & 216 & 118 \\
\hline CP3 & $\begin{array}{l}\text { Delegaciones } \\
\text { (busqueda) de nuevos } \\
\text { clientes }\end{array}$ & 83 & 47 & 157 & 236 & 123 \\
\hline CP4 & $\begin{array}{l}\text { Lograr/compartir } \\
\text { informacion del } \\
\text { marcado }\end{array}$ & 69 & 42 & 188 & 210 & 137 \\
\hline CP5 & $\begin{array}{l}\text { Hacer referencias } \\
\text { (recomendaciones) } \\
\text { para negocios cercanos }\end{array}$ & 76 & 36 & 173 & 231 & 130 \\
\hline CP6 & $\begin{array}{l}\text { Investigacion del } \\
\text { mercado conjunta. }\end{array}$ & 78 & 54 & 197 & 184 & 133 \\
\hline CP7 & $\begin{array}{l}\text { Desarrollo de nuevo } \\
\text { producto }\end{array}$ & 105 & 63 & 176 & 186 & 113 \\
\hline
\end{tabular}

\section{Ilustración 3 (Cooperación de marketing)}

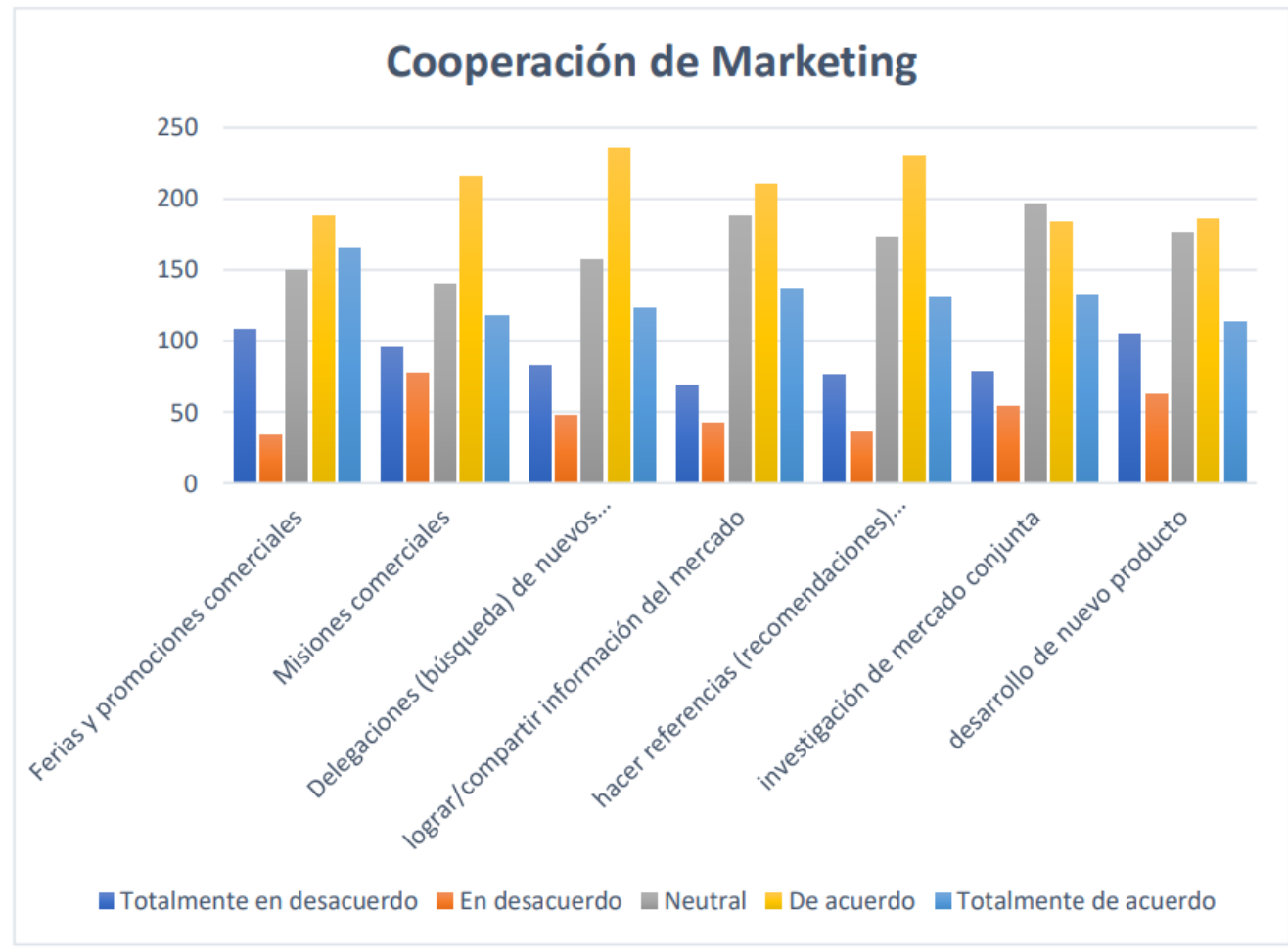


Tabla 4 (Intensidad de la internacionalización)

\begin{tabular}{|l|l|l|l|l|l|l|l|l|l|l|l|}
\hline \multicolumn{2}{|c|}{$\begin{array}{l}\text { INTENSIDAD DE } \\
\text { INTERNACIONALIZACIÓN }\end{array}$} & \multicolumn{3}{|c|}{ Bajo } & \multicolumn{3}{c|}{ Medioo } & \multicolumn{3}{c|}{ Alto } \\
\hline II1 & $\begin{array}{l}\text { EI numero de mercados } \\
\text { extranjeros (paises) que son } \\
\text { mercados objetivos de las } \\
\text { empresas de la asociación. }\end{array}$ & 156 & 63 & 35 & 39 & 66 & 60 & 42 & 52 & 74 & 59 \\
\hline II2 & $\begin{array}{l}\text { La participacion de las } \\
\text { exportaciones en las ventas } \\
\text { totales de productos de la } \\
\text { asociacion }\end{array}$ & 103 & 44 & 59 & 47 & 85 & 62 & 43 & 75 & 60 & 66 \\
\hline II3 & $\begin{array}{l}\text { El nuemro de acuerdos de } \\
\text { cooperacion formales con } \\
\text { socios extranjeros (otras } \\
\text { oficinas centrales, empresas, } \\
\text { entidades de I+D) }\end{array}$ & 152 & 75 & 38 & 61 & 59 & 54 & 50 & 55 & 58 & 40 \\
\hline II4 & $\begin{array}{l}\text { La cantidad de redes } \\
\text { internacionales en las que } \\
\text { participa la asociación. }\end{array}$ & 161 & 72 & 41 & 38 & 56 & 41 & 66 & 71 & 59 & 36 \\
\hline
\end{tabular}

\section{Ilustración 4 (Intensidad de la internacionalización)}

\section{Intensidad de internacionalización}

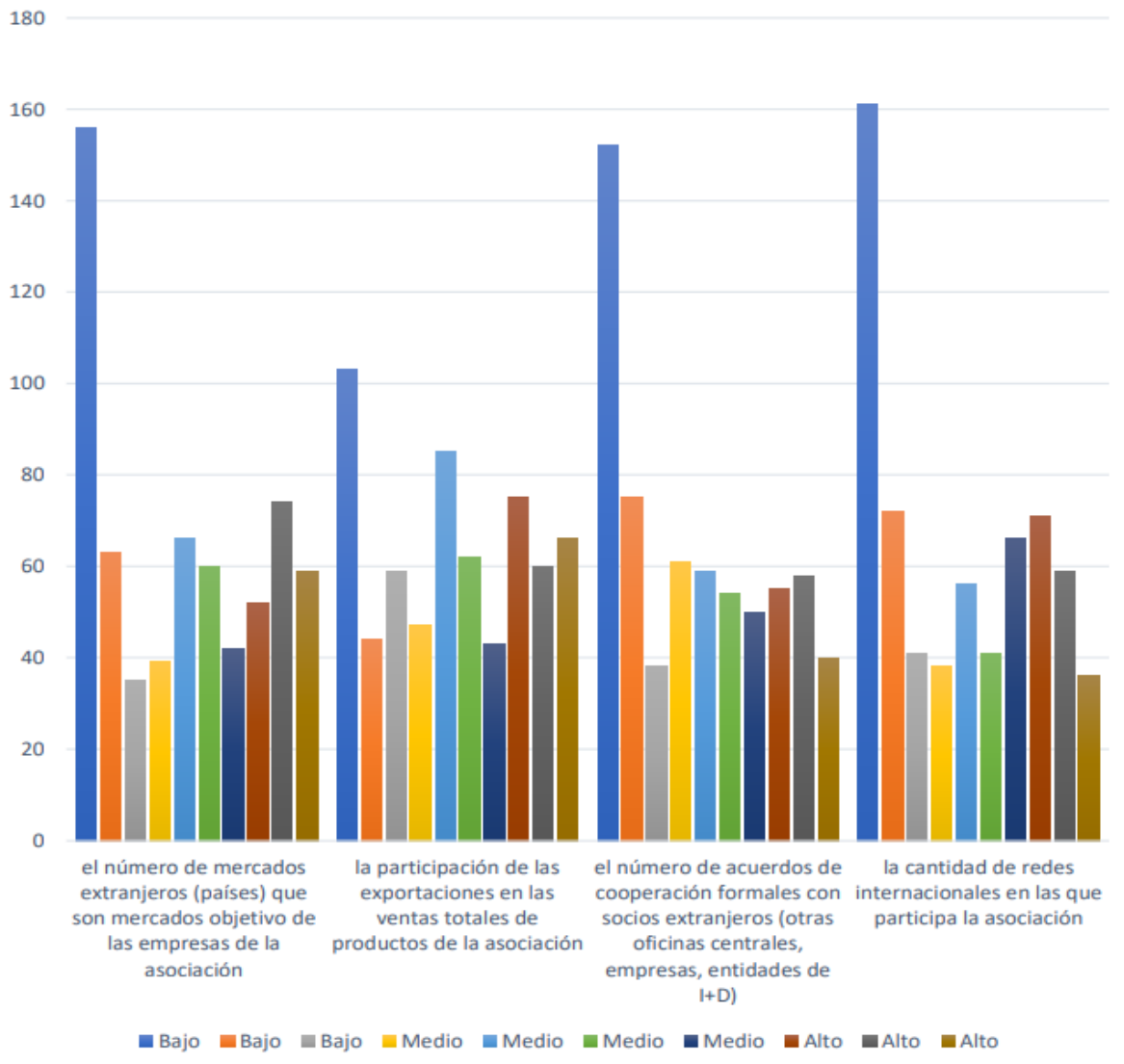




\section{Conclusiones}

El presente trabajo ha permitido conocer el desenvolvimiento de las asociaciones dentro del mercado, sus conocimientos e interés sobre una buena planeación de marketing, y el desarrollo de estrategias específicas que ayuden a su internacionalización; reconociendo que trabajar bajo el paraguas de una asociación, permite a las empresas llegar a otros mercados, promocionarse mejor y optimizar recursos. La información presentada junto con las hipótesis propuestas, dan paso a las siguientes conclusiones.

En primer lugar, las empresas se encuentran totalmente de acuerdo en plantear un proceso fundamental para el funcionamiento de la empresa, así como plantear estrategias que le permitan alcanzar sus objetivos empresariales, por otro lado, se encuentran de acuerdo en adquirir nuevos equipos y planear plantear objetivos de manera periódica. Realizar un análisis interno de las asociaciones enfocado a la creación de estrategias, contribuiría a conseguir los objetivos actuales y replantear los mismos, con la ayuda de la actualización de los equipos y maquinarias necesarios, pertenecientes a las empresas.

En segundo lugar, se debe desarrollar en un ámbito de negociación ganar - ganar, mediante buenas prácticas comerciales y leales con los asociados, permitiendo la ética en los negocios. En este punto las asociaciones mencionan que es importante crear alianzas con intereses estratégicos con competidores que se interesen en estrategias de asociación, cadenas o redes. Por lo tanto, es recomendable mejorar la relación actual con los aliados y buscar crear alianzas con empresas en otros mercados internacionales y así mismo en el mercado nacional.

En tercer lugar, las asociaciones necesitan nuevos clientes, así como hacer una investigación de mercado conjunta que permita el desarrollo de nuevos productos. De esta forma es importante investigar en el mercado posibles eventos, como ferias y promociones comerciales donde puedan ser partícipes, logrando posicionarse en la mente de los consumidores.

Por último, las asociaciones que exportan y aquellas que no, pero tienen la capacidad de exportar, dentro de la intensidad de internacionalización, mostraron que existe un número bajo de mercados extranjeros que son mercado objetivo de la empresas de la asociación; por otro lado, mencionan que su participación en ventas tienen un número pequeño en el mercado exterior, así como el número de acuerdos de cooperación formales con socios extranjeros y la cantidad de redes internacionales en las que participa la asociación son de intensidad baja. El aplicar estrategias de precios y promociones ayudaría a mejorar la relación con los socios internacionales actuales, de tal manera que se obtengan recomendaciones y así crecer en el mercado extranjero.

Una de las principales limitaciones del presente estudio es el ámbito geográfico que se considera una oportunidad para ampliar la investigación.

El presente trabajo es producto del grupo de investigación COMARK y de los estudiantes de V nivel de la asignatura de Marketing Comunitario de la Carrera de Mercadotecnia, en donde ejecutan las horas de prácticas de servicio comunitario. 


\section{Referencias}

Álvarez, Silvia Landin, Francisco Benítez-Luzuriaga, and Irene Sánchez-Gonzalez. "Análisis comparativo del crecimiento y participación del consumo de cacao ecuatoriano en Europa." Conference Proceedings. Vol. 1. No. 1. 2017.

Chiavenato, I., y Sapiro, A. (2017). Planeación estratégica. McGraw-Hill Interamericana.

Bustamante, C. (2017). Asociatividad como modelo para la internacionalización de los pequeños productores de banano en El Oro-Ecuador. Universidad de especialidades espíritu santo, pp. 1-25.

Orozco, R. V. (2017). El impacto del comercio del Banano. Revista AFESE.

Payares, O. B. (2018). Planeación estratégica y sostenibilidad corporativa. Conocimiento global. Vol.3 No. 1, pp. 50-55.

Carbajal, L. M. B., Tovar, L. A. R., y Zimmerman, H. F. L. (2017). Modelo de asociatividad en la cadena productiva en las Mipymes agroindustriales. Contaduría y administración, 62(4), pp. 1100-1117.

Vaca, L. F., Ronquillo, M. F., Fajardo, C. V., Ruiz, D. T., y Vaca, L. F. (2016). La Asociatividad Micro empresarial, como mecanismo productivo sostenible: Caso Cantón Milagro- $\quad$ Ecuador. Universidad Ciencia y Tecnología, 20(78).

Lobos, F. (2019). Evolución del marketing. Economìa y Sociedad, Vol 13.

Mosquera, J. (2018). La Historia y Evolución del Marketing. HISTORIA DEL MARKETING.

Alarcón, C. (2018). El marketing estratégico. Dominio de las ciencias, Vol 4.

Sukier, H., Hernandez-Fernandez, L., Portillo-Medina, R., Valle-Ospino, A., Garcia, M., y Garcia-Guiliany, J. (2018). Marketing estratégico: una mirada desde el contexto de la empresa familiar. Revista Espacios, 39(44).

Bustamante, C. (2017). Asociatividad como modelo para la internacionalización de los pequeños productores de banano en el oro-ecuador. Universidad de especialidades espíritu santo, pp. 1-25.

Betty Aguilar Echeverría y Erika Angulo Bennett (2017): "La asociatividad de las pymex como estrategia de internacionalización", Revista Observatorio de la Economía Latinoamericana, Ecuador, (octubre 2017).

Hoyos, O. (2019). Factores que impulsan la internacionalización en las pymes industriales del Eje Cafetero colombiano. Entramado, 15(1), 78-103. https://doi.org/10.18041/19003803/entramado.2.5597. 
Novoa, G. (2016). La Internacionalización de las Empresas en Colombia. Instituto Universitario ISSN: 2172-7856.

Andrade, E. (2017). Internacionalización de la mediana empresa familiar ecuatoriana: el rol de la orientación a la administración y flexibilidad estratégica del equipo gerencial. INNOVA Research Journal, 2(81), 350-337. ISSN 2477-9024.

Echeverría y Erika Angulo Bennett (2017): “La asociatividad de las pymex como estrategia de internacionalización”, Revista Observatorio de la Economía Latinoamericana, Ecuador, (octubre 2017).

Rave, E., Rubio, A., y Gutiérrez, S. (2018). Factores claves en la internacionalización de las pymes: Estudio empírico en el sector textil confección del área metropolitana del Valle de Aburrá Colombia. Espacios, 39(34), 18. ISSN 07981015.

Botello, H. (2014). Condiciones determinantes de la Internacionalización de las Empresas Industriales Latinoamericanas. Universidad Industrial de Santander Colombia. 41(). ISSN 0252-1865.

Ministerio de Agricultura, Ganadería, Acuicultura y Pesca, (2016). La Política Agropecuaria ecuatoriana Hacia el Desarrollo Territorial Rural Sostenible 2015-2025

Sánchez-González, I y Moscoso-Parra, A (2018). "Marketing, Comercio internacional y las TIC's-Marketing internacional." Machala: Universidad Técnica de Machala.

Zhuminaicela, J., Quevedo, J., y García, R. (2020). La producción de banano en la Provincia de El Oro y su Impacto en la agrodiversidad. Metropolitana de Ciencias Aplicadas, 3(3), 189-195. ISSN:2631-2662.

Burgos, O., López, R., y Ramírez, A. (2018). La revolución verde, el desarrollo Acuícola, la industria y la economía en Ecuador y Provincia de El Oro. Agroecosistemas, 6(2), 178184.

Diario EL COMERCIO, (2017). Obtenido de: https://www.elcomercio.com/actualidad/elorofincas-banano-exportacion.html.

Ministerio de Comercio Exterior, (2017). " Informe del Sector Bananero Ecuatoriano"

ESPOL, (2018). Obtenido de: Varela, H., Elizalde, B., Solórzano, S., y Vahttps://www.google.com/url?sa=t\&source=web\&rct=j\&url=http://www.espae.espol .edu.ec/wpcontent/uploads/2018/01/ei_acuicultura.pdf\&ved=2ahUKEwjm9pio_e3sAhWC1VkK HcAqB7EQFjAEegQIBhAB\&usg=AOvVaw1Hv8_EEeFL_e4NmwtX5LOb\&cshid=1 604667854274.

rela, G. (2017). Exportación de Camarón de la provincia de El Oro en el contexto del Tratado Comercial con la Unión Europea. Espacios, 61(38), 24. 


\section{Anexos}

Nos encontramos realizando un estudio sobre las asociaciones de la provincia de El Oro, y deseamos conocer su opinión. Los datos que nos proporcione serán tratados con total confidencialidad con el objetivo del bienestar empresarial de estos grupos. ¡Gracias!

PARTE 1. A continuación le mostramos 7 afirmaciones sobre la relación de conocimiento que tiene su empresa con otras. Indique su grado de acuerdo o desacuerdo con estas afirmaciones:

\begin{tabular}{|c|c|c|c|c|c|c|}
\hline \multicolumn{2}{|r|}{ COOPERACIÓN DE MARKETING } & \multicolumn{2}{|c|}{$\begin{array}{c}\text { Totalmente en } \\
\text { desacuerdo }\end{array}$} & Neutral & \multicolumn{2}{|c|}{$\begin{array}{c}\text { Totalmente de } \\
\text { acuerdo }\end{array}$} \\
\hline CP1 & Ferias y promociones comerciales & 1 & 2 & 3 & 4 & 5 \\
\hline$\overline{\mathrm{CP} 2}$ & Misiones comerciales & 1 & 2 & 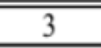 & 4 & 5 \\
\hline CP3 & Delegaciones (búsqueda) de nuevos clientes & 1 & 2 & 3 & 4 & 5 \\
\hline $\mathrm{CP} 4$ & lograr/compartir información del mercado & 1 & 2 & 3 & 4 & 5 \\
\hline CP5 & hacer referencias (recomendaciones) para negocios cercanos & 1 & 2 & 3 & 4 & 5 \\
\hline CP6 & investigación de mercado conjunta & 1 & 2 & 3 & 4 & 5 \\
\hline CP.7 & desarrollo de nuevo producto & 1 & 2 & 3 & 4 & 5 \\
\hline
\end{tabular}

PARTE 2. Sobre las diversas estrategias que tienen las empresas de la asociación. Indique su grado de acuerdo o desacuerdo con estas afirmaciones:

\begin{tabular}{|c|c|c|c|c|c|c|}
\hline \multicolumn{2}{|r|}{ PLANEACIÓN ESTRATÉGICA } & \multicolumn{2}{|c|}{$\begin{array}{c}\text { Totalmente en } \\
\text { desacuerdo }\end{array}$} & \multirow{2}{*}{$\frac{\text { Neutral }}{3}$} & \multicolumn{2}{|c|}{$\begin{array}{l}\text { Totalmente de } \\
\text { acuerdo }\end{array}$} \\
\hline PE1 & $\begin{array}{l}\text { La planeación es un proceso fundamental para el } \\
\text { funcionamiento de la empresa }\end{array}$ & 1 & 2 & & 4 & 5 \\
\hline PE2 & $\begin{array}{l}\text { Se plantean objetivos de manera periódica dentro de la } \\
\text { empresa }\end{array}$ & 1 & 2 & 3 & 4 & 5 \\
\hline PE3 & $\begin{array}{l}\text { Se plantean estrategias para alcanzar los objetivos } \\
\text { propuestos por la empresa }\end{array}$ & 1 & 2 & 3 & 4 & 5 \\
\hline PE4 & Adquisición de nuevos equipos & 1 & 2 & 3 & 4 & 5 \\
\hline
\end{tabular}


PARTE 3.

\begin{tabular}{|c|c|c|c|c|c|c|}
\hline \multicolumn{2}{|r|}{ ASOCIATIVIDAD } & \multicolumn{2}{|c|}{$\begin{array}{c}\text { Totalmente en } \\
\text { desacuerdo }\end{array}$} & \multirow{2}{*}{$\frac{\text { Neutral }}{3}$} & \multicolumn{2}{|c|}{$\begin{array}{l}\text { Totalmente de } \\
\text { acuerdo }\end{array}$} \\
\hline AS1 & $\begin{array}{l}\text { Las estrategias de negociación se desarrollan en } \\
\text { escenarios de ganar-ganar }\end{array}$ & 1 & 2 & & 4 & 5 \\
\hline AS2 & Se manifiesta voluntad de asociación & 1 & 2 & 3 & 4 & 5 \\
\hline AS3 & $\begin{array}{l}\text { Se establecen buenas prácticas comerciales y leales con } \\
\text { los asociados }\end{array}$ & 1 & 2 & 3 & 4 & 5 \\
\hline$\overline{\mathrm{AS} 4}$ & Hay ética en los negocios & 1 & 2 & 3 & 4 & 5 \\
\hline AS5 & Hay afectos con los empresarios de la competencia & 1 & 2 & 3 & 4 & 5 \\
\hline AS6 & Hay interés por alianzas estratégicas con competidores & 1 & 2 & 3 & 4 & 5 \\
\hline AS7 & $\begin{array}{l}\text { Conoce y le interesan estrategias de asociación, cadenas o } \\
\text { redes }\end{array}$ & 1 & 2 & 3 & 4 & 5 \\
\hline AS8 & $\begin{array}{l}\text { Indique cuál es el grado de importancia de asociarse para } \\
\text { su empresa }\end{array}$ & 1 & 2 & 3 & 4 & 5 \\
\hline
\end{tabular}


PARTE 4.

\begin{tabular}{|l|l|c|c|c|c|c|c|c|c|c|c|}
\multicolumn{3}{c}{ INTENSIDAD DE INTERNACIONALIZACIÓN } & \multicolumn{3}{c|}{ Bajo } & \multicolumn{7}{c|}{ Medio } \\
\hline III & $\begin{array}{l}\text { el número de mercados extranjeros (países) que son } \\
\text { mercados objetivo de las empresas de la asociación }\end{array}$ & 1 & 2 & 3 & 4 & 5 & 6 & 7 & 8 & 9 & 10 \\
\hline II2 & $\begin{array}{l}\text { la participación de las exportaciones en las ventas totales de } \\
\text { productos de la asociación }\end{array}$ & 1 & 2 & 3 & 4 & 5 & 6 & 7 & 8 & 9 & 10 \\
\hline II3 & $\begin{array}{l}\text { el número de acuerdos de cooperación formales con socios } \\
\text { extranjeros (otras oficinas centrales, empresas, entidades de } \\
\text { I+D) }\end{array}$ & 1 & 2 & 3 & 4 & 5 & 6 & 7 & 8 & 9 & 10 \\
\hline II4 & $\begin{array}{l}\text { la cantidad de redes internacionales en las que participa la } \\
\text { asociación }\end{array}$ & 1 & 2 & 3 & 4 & 5 & 6 & 7 & 8 & 9 & 10 \\
\hline
\end{tabular}

